\title{
Predicting the Risk for Angle Closure as Defined by the Shaffer System Using Anterior Segment Optical Coherence Tomography: A Simple Approach
}

\author{
Dan Samaha, OD, MSc \\ University of Montreal, \\ School of Optometry \\ Sébastien Gagné, MD, FRSC \\ Institut de l'œil des \\ Laurentides \\ Marie-Eve Corbeil, OD, MSc \\ University of Montreal, \\ School of Optometry
}

Pierre Forcier, OD, MSc University of Montreal, School of Optometry

\begin{abstract}
\section{PURPOSE}

To propose a simple non-invasive method for screening patients at risk for angle closure using anterior segment OCT.

\section{METHODS}

Scans of nasal and temporal iridocorneal angles in glaucoma suspect patients were performed using OCT. Upon identifying Schwalbe's line, the integrated caliper tool was used to draw a line to the nearest point of the iris to produce a measure 'S-I'. Gonioscopy was performed and angles graded according to Shaffer's classification to assess the correlation between both methods.
\end{abstract}

\section{RESULTS}

Thirty-four images were available for analysis. Spearman correlation coefficients between S-I anf gonioscopy grades were 0.81 for nasal and 0.77 for temporal quadrants respectively. Intraobserver ICC calculations demonstrated excellent reproducibility (0.98 and 0.99 for nasal and temporal angles) and excellent interobserver correlation (0.94 and 0.93). The diagnostic cutoff value of S-I for occludable angles was established at $330 \mathrm{~mm}$.

\section{CONCLUSION}

S-I measurement strongly correlates with gonioscopy and may be a suitable alternative for evaluating risk for angle closure.

\section{KEY WORDS:}

anterior chamber angle imaging, ASOCT, glaucoma, laser peripheral iridotomy, optical coherence tomography

Approximately 15 million people worldwide currently suffer from primary angle closure glaucoma (PACG) ${ }^{1}$ this disease constitutes the leading cause of medically and surgically irreversible blindness. This type of glaucoma is caused by the apposition of peripheral iris onto the trabecular meshwork, resulting in obstructing and impeding aqueous humor outflow through the anterior chamber angle. Pupillary block, which accounts for 90\% of PACG cases, ${ }^{2}$ is caused by apposition of the iris onto the anterior face of the lens, which restricts aqueous humor flow from the posterior to anterior chamber. The accumulation of aqueous humor in the posterior chamber causes anterior bowing of the iris and eventual angle closure. This mechanism can occur acutely, intermittently or chronically. The mean prevalence for PACG worldwide is currently estimated at $0.69 \%{ }^{1}$

In the last 40 years, numerous risk factors have been identified, such as hyperopic eyes, small corneas and high iris insertion, but observation of the irido-corneal angle through gonioscopy remains the clinical standard when screening for this disease. Although easily carried out, gonioscopy presents its limitations: the necessity for direct contact with an anesthetized cornea, 
skewed results caused by pressure on the cornea or too much ambient light artificially opening the angle. Moreover, multiple studies have demonstrated great variability in gonioscopic findings among experienced observers. ${ }^{3-5}$

With the advent of optical coherence tomography (OCT) in ophthalmology, ${ }^{6}$ its use was previously exclusive to the study of the posterior segment (retina). Nowadays rapid acquisition of high definition anterior segment images is possible, all the while eliminating the need for corneal contact.?

Multiple studies have already described different approaches for quantitatively defining the irido-corneal opening, ${ }^{7-10}$ while using the scleral spur as their anatomical landmark. Being non-identifiable $20-30 \%$ of the time, ${ }^{8}$ authors quickly turned towards Schwalbe's line as their new anatomical reference; ${ }^{9-10}$ which can be visualized and identified in more than $95 \%$ of the anterior segment OCT images. Current models of measure compute anterior segment characteristics such as Angle Opening Distance (AOD), Trabecular-Iris-Surface-Area (TISA) and Schwalbe's line Angle Opening Distance (SL-AOD) which have been shown to have good inter and intraobserver reliability. However, these measurements still rely on observer caliper manipulations on several image points which are potentially subject to manipulation errors. Although some OCT instruments can readily compute these measurements, many other devices cannot, requiring exporting the images onto another platform, increasing the time needed for image analysis.

In this study, we suggest a simple approach using anterior segment optical coherence tomography, or AS-OCT imaging, which would allow one to rapidly image and quantify the irido-corneal opening by determining the minimal distance between Schwalbe's line and iris. A single line traced from iris to Schwalbe's line could potentially be simpler to draw than exporting an image and placing a multi-point caliper on the anterior segment angle. This tool could be used as a quicker and non-invasive screening method, for patients in which angle closure is suspected.

\section{METHODS}

\section{Subjects}

In this prospective study, forty Caucasian subjects (22 females, 18 males) were recruited from the glaucoma clinic at the Institut de l'Oeil des Laurentides (IOL), in Boisbriand (Quebec). The patients were seen for the first time at the Institute and had been referred by their respective optometrist as glaucoma suspects, whether because they had elevated intra-ocular pressure, suspicious cup-to-disc ratio, etc. Written informed consent was obtained from all participants, after the study had received approval from Montreal University's ethics committee review board (Certificate \#13-029-CERES-D), in accordance to the tenets of the Declaration of Helsinki.

All subjects included underwent ophthalmic examination, including visual acuity, slit-lamp biomiscroscopy, Goldmann applanation tonometry and darkroom gonioscopy. Subjects with a history of previous intraocular surgery, penetrating trauma, pigment dispersion syndrome or any corneal opacities or abnormalities that precluded AS-OCT imaging were excluded. Those having previously undergone laser peripheral iridotomy (LPI) were not excluded.

An experienced glaucoma specialist (SG) performed all the aforementioned exams, and used a G4 Volk 4-mirror gonioscope in a darkened examination room. Using a $1 \mathrm{~mm}$ wide and $7 \mathrm{~mm}$ high slit beam, vertically offsetting the light to enable corneal wedge visualization and avoiding light exposure through the pupil, grading was recorded for 4 angle quadrants (superior, inferior, nasal and temporal) in both eyes using the Shaffer grade classification. According to the American Academy of Ophthalmology (AAO) this classification is the most commonly used. Grading properties were as follows: grade 4 for a wide-open angle (35-45 degrees); grade 3 for a wide-open angle (20-35 degrees); grade 2 was used to denote an angle with a possible risk of closure (20 degrees); a quadrant was graded at 1 or less (10 degrees or less) to signify a high risk of closure. Indentation gonioscopy was performed to verify the presence or absence of peripheral anterior synechiae (PAS). Patients with evidence of PAS were excluded.

\section{ANTERIOR CHAMBER AND ANGLE IMAGING}

\section{AS OCT}

Imaging of the angle was performed on undilated pupils, with a Fourier-domain (spectral) high definition optical coherence or SD-OCT system (Spectralis, Heidelberg Engineering, Germany). The apparatus utilizes an 820nm wavelength light source and has a scanning speed of 40,000 A-scans/second. Image lateral resolution can reach 7 microns per pixel. An anterior segment objective lens was mounted for anterior segment imaging. All scans were performed under uniform dim illumination of 3.5 lux, as measured with a luxmeter (Sekonic model L-308DC, DigiCineMate, North White Plains, NY). Scans were acquired with the 'angle to angle' module to image both nasal and temporal angles at the same time (Figure 1), on the same image. A fixation target was utilized to properly guide the gaze of the subject into primary position. Only images of nasal and temporal quadrants were acquired, due to technical difficulties in scan acquisition of the superior and inferior quadrants. 
Figure 1: Anterior segment Imaging using Spectralis optical coherence tomography; nasal and temporal angles in a single capture.

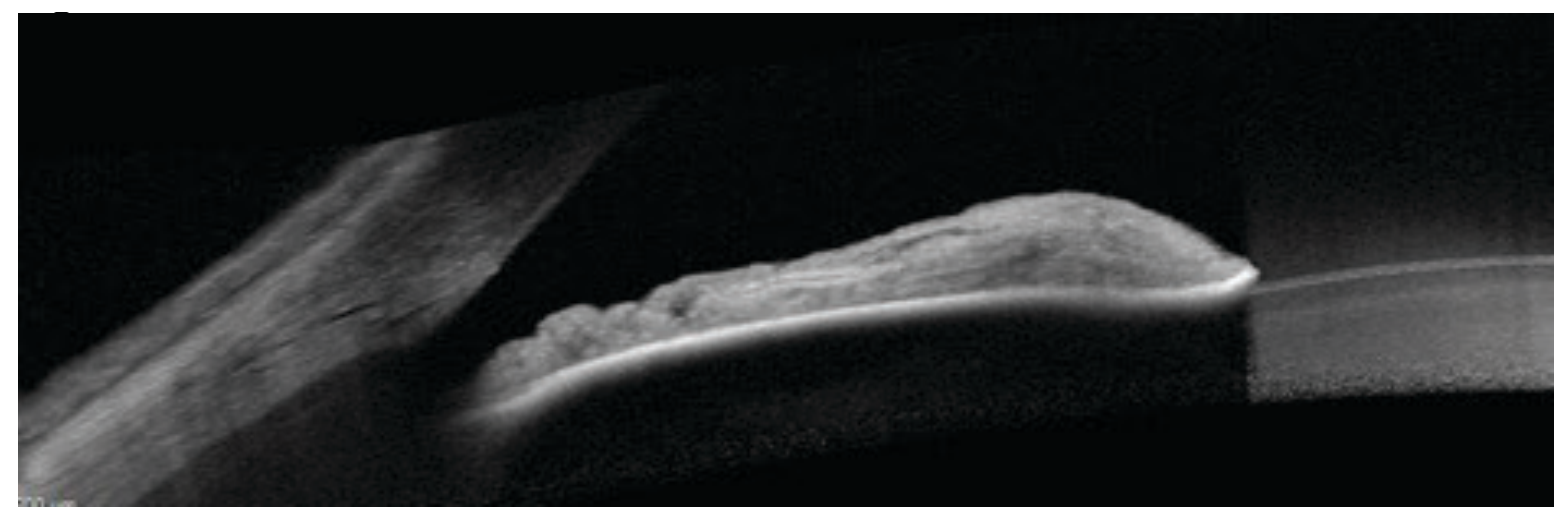

Quantitative assessment: S-I

Images presenting artifacts hindering identification of anatomical landmarks (Schwalbe's line) were excluded. Two observers (DS, SG) independently carried out the measurements. After identifying Schwalbe's line, a line was drawn between $S$ and the closest point of the iris using the Spectralis line tracer integrated tool. The measurement was instantaneously displayed in microns and the observer could therefore readily identify the smallest measure possible. We called this measure S-I (Figure 2). Observer DS re-graded the randomly reordered images two weeks later.

Figure 2: Anterior chamber angle in an anterior segment optical coherence tomography line scan image. (A) One can clearly identify Schwalbe's line as the little indentation signifying the termination of the corneal endothelium. (B) S-I measurement using the integrated measurement tool. The distance is automatically displayed and shown here as $311 \mu \mathrm{m}$.

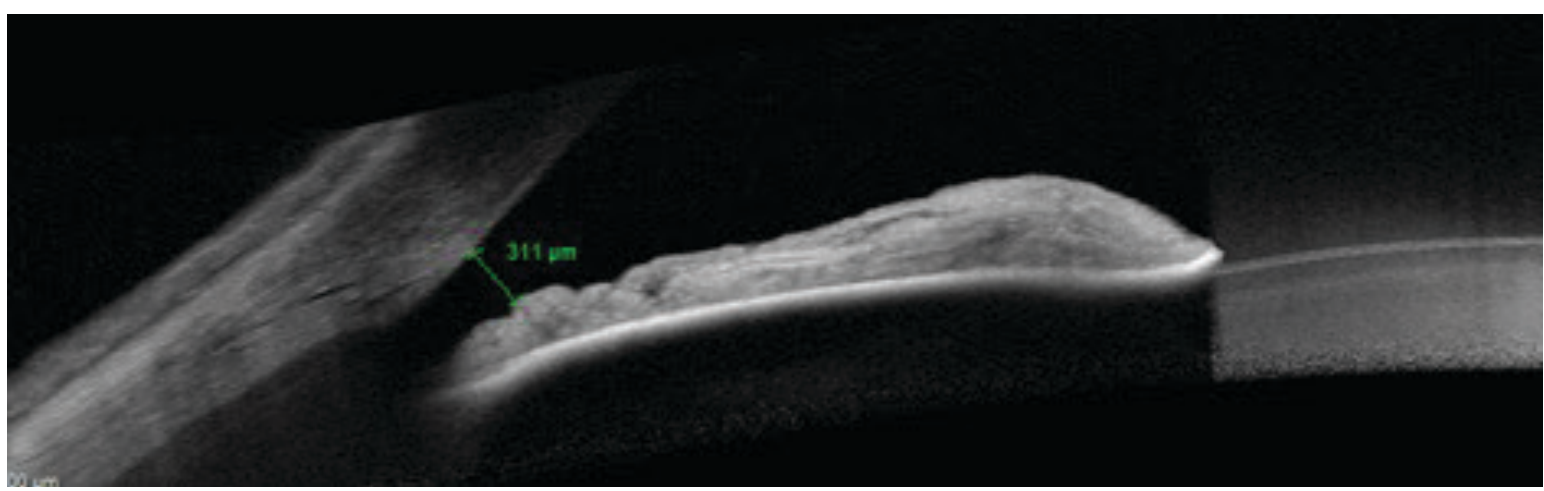

\section{Statistical analysis}

One eye from each subject was randomly selected for data analysis. Statistical analyses were performed using SPSS version 25 (developed by SPSS, Chicago, IL). The chi-square test was used to compare categorical data. ANOVA and Tukey Kramer tests were used to assess the difference between S-I means for each gonioscopic grade to evaluate whether their differences were significant, S-I being quadrant-specific (nasal and temporal). An intraclass coefficient correlation analysis (ICC) was used to assess inter and intraobserver reproducibility. Finally, Spearman's Rho coefficient was used to evaluate the strength of association between S-I and gonioscopic grading.

\section{RESULTS}

All patients were Caucasian. The mean age (Standard Deviation SD) was 59.7 years (7.67, range 41-75) and average spherical equivalent was $+0.75 \pm 1.50 \mathrm{D}$. Eight images were excluded owing to poor quality, leaving 32 images available for analysis (14 right eyes and 18 left eyes). There were no significant differences in mean age and gender for all included subjects (using Chi -square analysis, data not shown). Seventeen patients were found to have gonioscopically occludable angles (Shaffer grade 1 or less), five of which had already previously undergone peripheral laser iridotomy. The correlation between S-I measurements and gonioscopic grading was found to be statistically significant (Spearman $r=0.81$ and 0.79 for the nasal and temporal quadrants respectively, $\boldsymbol{p}<0.05$ ). 
Table 1 demonstrates results from Tukey Kramer HSD testing, illustrating the differences between means of S-I for each gonioscopic grade for nasal and temporal quadrants respectively. Positive values indicate a pairing that is significantly different. For example, looking at table 1, the first line compares the mean S-I value for a Shaffer grade 4 with the mean S-I value of all the other Shaffer grades. One can observe that the mean S-I value for a grade 4 is significantly different from S-I means from Shaffer grades 2, 1 and 0. Figure 3 graphically represents those results, accompanied by the comparison circles.

Table 1: Comparison between means using Tukey-Kramer HSD test (OCT S-I N vs Gonio grade)

\begin{tabular}{|c|c|c|c|c|c|}
\hline $\begin{array}{c}\text { SHAFFER } \\
\text { GRADE }\end{array}$ & 4 & $\mathbf{3}$ & $\mathbf{2}$ & $\mathbf{1}$ & $\mathbf{0}$ \\
\hline 4 & $\mathrm{X}$ & -14.1978 & 28.342 & 126.3621 & 106.3621 \\
\hline 3 & -14.1978 & $\mathrm{x}$ & 5.988 & 19.90661 & 1.64431 \\
\hline 2 & 28.3242 & 5.988 & $\mathrm{x}$ & -3.427 & -9.621 \\
\hline 1 & 126.8587 & 19.90661 & -3.427 & $\mathrm{x}$ & -103.715 \\
\hline 0 & 106.3621 & 1.64431 & -9.621 & -103.715 & $\mathrm{x}$ \\
\hline
\end{tabular}

*Positive values show pairs of means that are statistically different

\begin{tabular}{|ccc|}
\hline GRADE & & AVERAGE \\
\hline 4 & A & 451.00 \\
\hline 3 & A B & 354.60 \\
\hline 2 & B C & 301.71 \\
\hline 1 & C & 232.81 \\
\hline 0 & C & 226.25 \\
\hline
\end{tabular}

Levels not connected by the same letter are largely different

Figure 3: Comparison of OCT S-I Nasal means with gonioscopic grading using Tukey-Kramer HSD analysis. The Younen index was used to determine the cutoff value for $S$-I between open angles and occludable angles (red line).

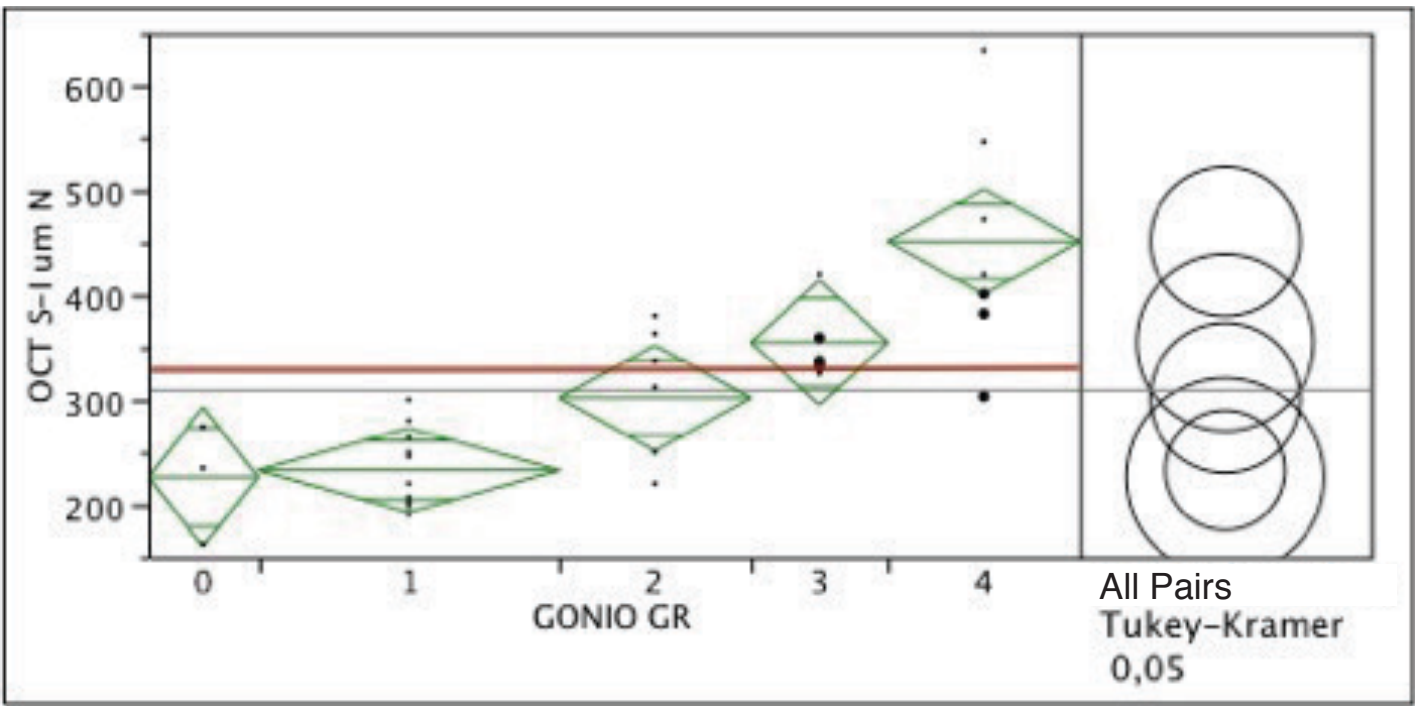


Interobserver ICC calculations demonstrated excellent reproducibility using ICC analysis $(0.94$ and $0.93, \mathrm{p}<0.001$ for nasal and temporal quadrants respectively). Using the same method, the analysis also revealed excellent intraobserver reproducibility ( 0.98 and $0.99, \mathrm{p}<0.001$ for nasal and temporal quadrants respectively).

DISCUSSION

This study aimed to devise a new, simple parameter to quantify the risk of angle closure, using Schwalbe's line as identified on anterior segment OCT imaging. Devoid of the need to transfer images on some of the available OCT instruments, use complex mathematical logarithms, or manipulate several caliper points on acquired images, our results demonstrated an excellent correlation between our S-I measurement and standard gonioscopic grading. This simple quantitative measure can be used to rapidly screen and monitor patients in whom angle closure is suspected while removing the necessity to topically anesthetize and create contact with the cornea of the patient. Moreover, this technique requires less operator manipulations to compute when compared with methods such as TISA and AOD measurements and eliminates the need for image transferring.

With the advent of high resolution imaging of the anterior chamber angle, visualization of SL is now more readily achieved; it was identified in $94 \%$ of analyzable images, a finding similar to previous studies. ${ }^{10,15}$ Other researchers have used the Sclera Spur or Trabecular Meshwork as the anatomical landmarks for the measurement of their parameters. ${ }^{16-17}$ A major limitation of using such landmarks is that they are only identifiable on 70-80\% of HD-OCT images. Thus, our findings showed that our parameter using SL as the anatomical landmark may be more suitable in assessing irido-corneal angles with respect to OCT imaging.

Figure 3 is a graphic illustration of the Tukey-Kramer HSD test results for the nasal quadrant. The results are fairly identical to the temporal quadrant (results not shown). The comparison circles of S-I values corresponding to gonioscopic grades 3 and 4 are significantly separated from S-I values corresponding to gonioscopically occludable angles. The red line displays the cutoff value for S-I $(330 \mathrm{~mm})$ between wide-open angles and those at risk for closure, as defined by ROC analysis and subsequent identification of Younen's index (table not shown).

One of the main limitations of this study is the lack of acquisition of HD-OCT images for the superior and inferior quadrants, owing to technical difficulties (eyelids and the need for a speculum). It is possible that the pattern of visibility of anatomical landmarks in those quadrants is different. Secondly, with HD-OCT imaging, an external fixation light must be used to properly position the eye of the subject and image the horizontal quadrants. The fixation pattern or position of the fixation light have yet to be standardized. Furthermore, all subjects in the study were Caucasian; as angle closure is more prevalent in other ethnicities, such as Asians, African-Americans and Eskimos, repeating the study on such groups might prove very useful to further characterize S-I across different populations. Finally, the relatively small sample size poses as another potential limitation.

\section{CONCLUSION}

Primary angle closure glaucoma is a sight-threatening disease. Current methods of screening include gonioscopy and UBM, both of which make surface contact with the eye and are difficult to master. Anterior segment imaging using HD-OCT is a promising and non-invasive method, but remains unstandardized in the screening of occludable angles. Results using our novel, and for some users, simpler measurement parameter, suggest S-I may be potentially useful in rapidly and easily quantifying the risk for angle closure. A cutoff value between open and closed irido-corneal angle was also provisionally provided $(330 \mathrm{~mm})$. Further studies should be elaborated to answer the aforementioned limitations. $\bullet$

\section{Corresponding author name and e-mail:}

Dan Samaha, dan.samaha@umontreal.ca, (514) 880-3266

No grant or financial compensation was received for the realization of this paper. 


\section{REFERENCES}

1. Quigley HA, Broman AT. The number of people with glaucoma worldwide in 2010 and 2020. Br J Ophthalmol 2006; 90: 262-7.

2. Patel K, Patel S. Angle-closure glaucoma. DM 2014; 60: 254-62.

3. Foster PJ, Devereux JG, Alsbirk PH, et al. Detection of gonioscopically occludable angles and primary angle closure glaucoma by estimation of limbal chamber depth in Asians: modified grading scheme. Br J Ophthalmol 2000; 84: 186-92.

4. Friedman DS, He M. Anterior chamber angle assessment techniques. Surv Ophthalmol 2008; 53: 250-73.

5. Schirmer KE. Gonioscopy and artefacts. Br J Ophthalmo 1967; 51: 50-3.

6. Huang D, Swanson EA, Lin CP, et al. Optical coherence tomography. Science 1991; 254: 1178-81.

7. Radhakrishnan S, Rollins AM, Roth JE, et al. Real-time optical coherence tomography of the anterior segment at $1310 \mathrm{~nm}$. Arch ophthalmol 2001; 119: 1179-85.

8. Leung CK, Li H, Weinreb RN, et al. Anterior chamber angle measurement with anterior segment optical coherence tomography: a comparison between slit lamp OCT and Visante OCT. Invest Ophthalmol Vis Sci 2008; 49: 3469-74.

9. Muller M, Dahmen G, Porksen E, et al. Anterior chamber angle measurement with optical coherence tomography: intraobserver and interobserver variability. J Cataract Refract Surg 2006; 32: 1803-8.

10. Qin B, Francis BA, Li Y, et al. Anterior chamber angle measurement using schwalbe's line with high resolution fourier-domain optical coherence tomography. J Glaucoma 2013; 22: 10.1097/ IJG.0b013e318264b921

11. Dinc UA, Oncel B, Gorgun E, et al. Assessment of anterior chamber angle using Visante OCT, slit-lamp OCT, and Pentacam. Eur J ophthalmol 2010; 20: 531-7.
12. Doors M, Cruysberg LP, Berendschot TT, et al. Comparison of central corneal thickness and anterior chamber depth measurements using three imaging technologies in normal eyes and after phakic intraocular lens implantation. Graefes Arch Clin Exp Ophthalmol 2009; 247: 1139-46.

13. Lange S, Haigis W, Grein HJ, et al. Comparison of different optical techniques for determination of the dimensions of anterior ocular segment. Klin Monbl Augenheilkd 2009; 226: 485-90.

14. Rossi GC, Scudeller L, Delfino A, et al. Pentacam sensitivity and specificity in detecting occludable angles. Eur J ophthalmol 2012; 22: 701-8.

15. Wong HT, Lim MC, Sakata LM, et al. High-definition optical coherence tomography imaging of the iridocorneal angle of the eye. Arch ophthalmol 2009; 127: 256-60.

16. Liu S, Li H, Dorairaj S, et al. Assessment of scleral spur visibility with anterior segment optical coherence tomography. J Glaucoma 2010; 19: 132-5.

17. Sakata LM, Lavanya R, Friedman DS, et al. Assessment of the scleral spur in anterior segment optical coherence tomography images. Arch ophthalmol 2008; 126: 181-5.

18. Antoniazzi E, Pezzotta S, Delfino A, et al. Anterior chamber measurements taken with Pentacam: an objective tool in laser iridotomy. Eur J ophthalmol 2010; 20: 517-22.

19. Kurita N, Mayama C, Tomidokoro A, et al. Potential of the pentacam in screening for primary angle closure and primary angle closure suspect. J Glaucoma 2009; 18: 506-12.

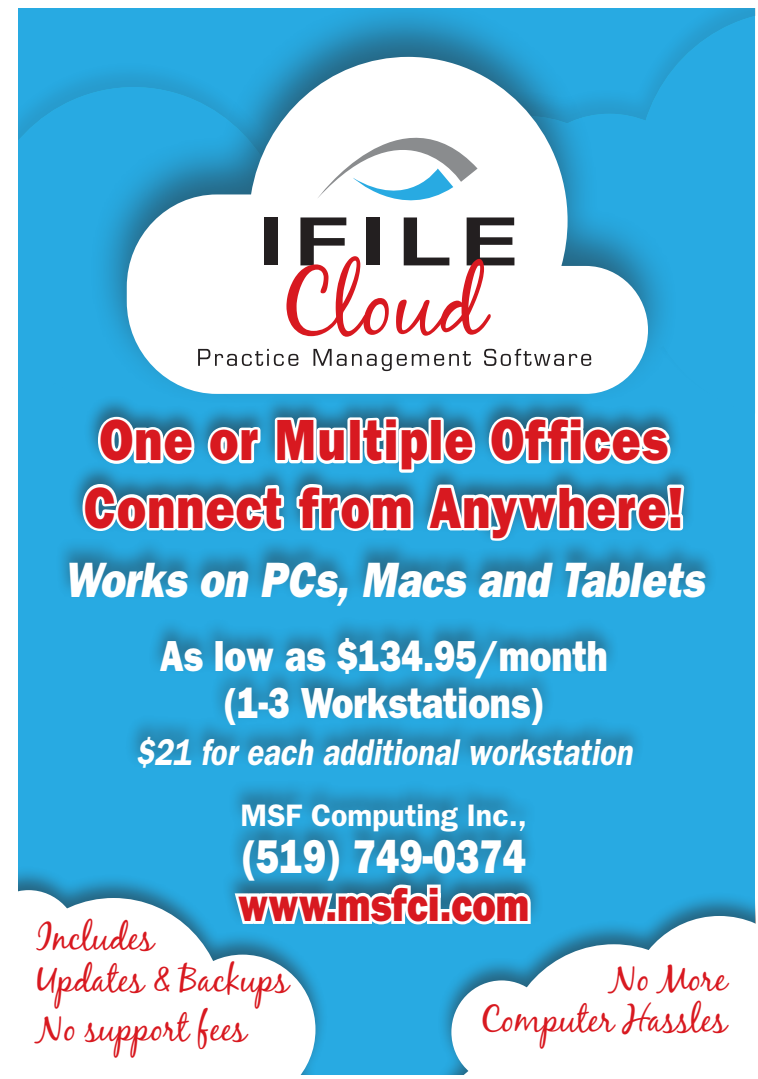

